\title{
Campus numériques français : pertinence des notions de réussite ou d'échec
}

French virtual campuses: the relevance of their success or failure

\section{Élisabeth Fichez}

\section{CpenEdition}

Journals

Édition électronique

URL : http://journals.openedition.org/edc/567

DOI : $10.4000 /$ edc. 567

ISSN : 2101-0366

Éditeur

Université Lille-3

Édition imprimée

Date de publication : 1 décembre 2007

Pagination : 49-71

ISBN : 978-2-9514961-8-7

ISSN : $1270-6841$

Référence électronique

Élisabeth Fichez, "Campus numériques français : pertinence des notions de réussite ou d'échec »,

Études de communication [En ligne], Numéro spécial | 2007, mis en ligne le 01 octobre 2009, consulté le 30 avril 2019. URL : http://journals.openedition.org/edc/567 ; DOI : 10.4000/edc.567

Ce document a été généré automatiquement le 30 avril 2019.

(c) Tous droits réservés 


\title{
Campus numériques français : pertinence des notions de réussite ou d'échec
}

French virtual campuses: the relevance of their success or failure

\author{
Élisabeth Fichez
}

1 Notre propos va être dans cet article d'approfondir le problème qui a surgi pour nous à la fin de l'étude menée dans le cadre de l'ERTe, à savoir : que dire en définitive du campus Cultura retenu comme terrain de notre étude de cas? Cela a-t-il un sens de parler à son propos, et plus largement à propos des autres campus, de réussite ou d'échec ${ }^{1}$ ? Pourquoi cette question, apparemment simple, ouvre-t-elle sur une certaine perplexité?

2 C'est le souhait de pouvoir répondre à une telle question de manière ni superficielle, ni naïve, qui nous a motivée à en faire un objet d'analyse, lequel, nous l'avons rapidement compris, allait nous amener à un retour réflexif distancié sur l'étude menée dans l'ERTe pour caractériser entre autres la nature de notre démarche et son rapport à une dimension évaluative.

3 Soyons d'emblée très claire sur la posture de recherche de cette équipe : elle n'était pas dans une problématique d'évaluation au sens où il se serait agi d'une recherche commanditée en vue d'évaluer les expériences, ou d'une recherche-action, un autre « genre » sur lequel nous allons revenir ci-dessous. La raison de fond qui a motivé les études est, comme cela est rappelé dans l'introduction à ce numéro, une interrogation sur les conditions de pérennisation de ces expériences (en quoi elles pouvaient constituer des modèles tenables dans la durée) en prenant le problème sous deux angles privilégiés : les conditions socio-économiques et les conditions socio-organisationnelles de pérennisation. La décision de focaliser notre attention sur ces deux types de conditions peut paraître discutable s'agissant de dispositifs ou de systèmes de formation: en effet, régulièrement, des études statistiques nous rappellent que des modèles qui tiennent dans la durée (par exemple les premiers cycles universitaires) peuvent être déficients sur le plan pédagogique ou sur celui de leur « productivité » en terme de réussite aux diplômes. 
Il y aurait donc une condition tout aussi importante à prendre en considération, à savoir la qualité du dispositif de formation lui-même et sa pertinence par rapport au public d'apprenants. En fait, la réalité concernée est un ensemble systémique et les niveaux d'analyse des conditions favorisantes sont multiples: macro (le contexte sociétal d'existence), méso (contexte institutionnel et inter-institutionnel des projets), micro (les dispositifs de formation mis en place). Nous nous sommes clairement situés au niveau «méso », l'argument justifiant notre choix étant que nous nous trouvions devant des formes socio-organisationnelles inédites (un des critères à satisfaire pour être retenu était la création de "consortium d'établissements " publics et privés) à la recherche de leur mode d'équilibre financier et il qu'y avait donc bien, sur le plan de la recherche, un terrain d'investigation (et des terrains pratiques) à défricher. Nous ne nous sommes en rien interdit pour autant, d'observer ce qui se passait au niveau micro sur le plan des dispositifs pédagogiques en croisant des matériaux issus de diverses sources.

Il est donc bien clair que notre dispositif d'étude et de recherche n'a pas la prétention d'être une " évaluation ", qu'il ne peut davantage prétendre à une approche systématique de la réalité étudiée et que, en outre, bien que bâti sur une grille d'enquête commune, il n'a pas requis une uniformité dans l'usage de cette grille par les différents enquêteurs. Nous proposons donc de reformuler le "possible" de notre propre analyse en ces termes : qu'est-ce que le prisme d'observation que nous avons choisi de mettre en œuvre collectivement, réfracté de manière singulière sur chaque terrain par les mises en œuvre individuelles (ou par binômes de chercheurs), permet de dire du succès ou de l'insuccès des projets?

5 Reste à préciser comment nous pouvons construire l'objet de l'analyse ainsi délimité et méthodologiquement justifié. Ce qui nous intéresse personnellement, en tant que spécialiste des sciences de la communication, c'est de concevoir les objets étudiés - à savoir les 5 campus numériques français relevant des appels à projets ministériels - en tant que projets de dispositifs de formation médiatisés liés à des intentions innovantes aussi bien de la part des responsables politiques que des acteurs qui ont tenté de leur donner corps sur le terrain ${ }^{2}$. Dès lors, il conviendra d'identifier ces intentions innovantes et de nous interroger sur ce qu'il nous semble en être advenu au terme d'une période limitée d'observation (2003-2005) en termes de tendances plus que de réalités stabilisées.

\section{"Mesurer des réussites ou des échecs »: problématisation et démarches méthodologiques}

6 Il nous parait important de revenir dans le premier temps de cette contribution sur la problématique en jeu et sur la façon dont notre étude se situe par rapport à d'autres types de démarches pour en traiter.

\section{La complexité des questions en jeu}

7 L'idée d'apprécier « des réussites ou des échecs » à propos de projets tels que ceux qui nous concernent pose, on l'a déjà entrevu, de nombreuses questions.

8 La première série d'entre elles renvoie ainsi à la diversité des niveaux auxquels une telle appréciation peut s'effectuer, étant entendu que, dans chacun de ces niveaux, des indicateurs liés à des rubriques tout aussi diverses sont à construire. Ainsi, s'agissant des 
regroupements partenariaux, on peut constater des situations d'échec au fil du temps qui fragilisent le dispositif de formation, mais qui pour autant ne l'empêchent pas d'exister et d'être considéré comme une réussite au moment où l'observation a lieu. De même un choix stratégique peut avoir conduit, dans la conception/mise en oeuvre du dispositif de formation (et donc de manière interne au niveau micro) à faire des ressources multimédias une réussite, et de l'accompagnement tutoral un échec (ou un semi-échec), parce que le coût élevé des ressources n'a pas permis de budgéter correctement ce poste de dépense dont l'importance a été au départ sous-estimée. La dimension systémique est donc primordiale et, s'il n'est pas possible dans telle ou telle démarche d'étude de l'envisager comme telle, une grande prudence s'impose dans les jugements portés pour ne pas ériger en réussite ou échec global ce qui ne l'est que de manière partielle, voire ponctuelle. Il est difficile aussi d'apprécier de quel poids, dans la pérennisation de l'expérience, tel ou tel facteur va être affecté par rapport à tel autre. Ainsi, Viens et Peraya (2004) font-ils remarquer à juste titre que "le succès ou l'insuccès d'un projet ne peuvent se mesurer à la seule qualité du dispositif de formation entendu strictement au sens de 'formation médiatisée' [...] ou de cours en ligne. A terme, l'évolution du profil de compétences des différents acteurs, l'évolution de leur pratique, doivent être considérées comme des facteurs de stabilisation et de pérennisation de l'innovation plus sûrs que la qualité d'un cours ou d'un logiciel distribué via le réseau $»^{3}$.

Une seconde série de questions tourne autour du sens et des enjeux d'une évaluation en termes de succès ou d'échec: Qui évalue? Pour qui ? Avec quels objectifs? Selon quelles méthodes? Nous approfondirons cet ensemble de questions en l'illustrant ci-dessous par trois types d'études différentes autour des campus numériques : s'il est clair par exemple que chacune d'elles vise un retour d'effets en direction des acteurs de terrain, la méthode d'intervention auprès de ceux-ci et le mode de travail avec eux sont très éloignés selon qu'il va s'agir d'une étude objectivante menée par des acteurs non impliqués dans la conduite du projet (cas 1 et 3), ou d'une approche participative qui implique analystes et acteurs directs comme dans une recherche-formation (cas 2).

Troisième et dernière série de questions (sans prétendre les avoir toutes inventoriées) : celles liées à la temporalité des projets, rapportée à la temporalité de l'observation/ évaluation. Même dans le cas d'observations relativement longues (les nôtres se sont étendues sur une période de deux ans environ), ces deux temporalités ne se recouvrent pas : un projet qui apparaît sur le mode de la réussite (ou inversement de l'échec) dans le temps de l'observation peut changer de statut hors des bornes de ce temps, soit en amont lorsqu'il était en creux dans un projet relativement proche ${ }^{4}$, soit lorsqu'il renaît en aval dans un autre contexte.

\section{Trois types de démarches d'étude concernant les campus numériques}

11 Avant de nous focaliser sur notre étude en propre, nous la situerons par rapport à deux autres démarches d'étude dans le domaine des campus numériques qui permettront d'illustrer de façon plus concrète la problématisation que nous venons d'esquisser et de répondre en particulier au deuxième ensemble de questions. 


\section{Des enquêtes objectivantes commanditées à des organismes spécialisés extérieurs au champ professionnel de l'enseignement supérieur}

Ce sont sans doute des études qui correspondent le mieux à ce que chacun a comme représentation classique d'une évaluation formelle commanditée. Ainsi, en France, divers cabinets ou instituts (l'institut IPSOS, ALGORA - spécialisés dans le champ précis de la FOAD -, la société de conseil « Éducation et Territoires »...) ont été sollicités par le MEN ou par les acteurs directs de tel ou tel campus, pour une évaluation soit de grande envergure (cf. celle d'IPSOS, Dupuis, Schmitt, Galissi et Robion, 2003), soit portant sur des aspects plus ponctuels (cf. par exemple celles d'Éducation et Territoires) $)^{5}$. Nous arrêtant sur celle d'IPSOS, nous dirons qu'elle a une visée clairement systémique en prenant comme champ l'ensemble des 64 campus censés être en activité à l'époque et en travaillant quantitativement et qualitativement sur trois grands axes correspondant aux trois niveaux que nous avons repérés: les effets sur l'offre nationale (meilleur équilibre territorial pour l'accès aux formations) et à l'international (en particulier la francophonie); les effets sur les organisations impliquées et notamment le caractère pérenne des projets; l'efficacité des dispositifs mis en place. Les 3 grandes catégories d'interlocuteurs interviewés et leur nombre élevé confirment cette visée systémique : des acteurs partenaires de l'opération (Direction de l'Enseignement Supérieur, Direction de la Technologie, DATAR, AUF, CNED, Edufrance, Conseils régionaux...), des chefs de projet et différents types d'acteurs au sein des établissements (64 chefs de projets, 23 Secrétaires Généraux d'université, 31 responsables d'établissement, 31 responsables techniques), les apprenants (278, usagers de 24 campus) et les enseignants ( 45 enseignants-auteurs et 45 enseignants-tuteurs). En ce qui concerne la temporalité de l'enquête, celle-ci a été menée en 4 «lots» d'avril à juin 2003, sous forme donc d'une prise de données ponctuelle.

Quant au sens et aux enjeux d'une telle enquête objectivante, il faut, pour les cerner, resituer la commande du Ministère dans le contexte « sociétal » des politiques publiques et gouvernementales de la période 2002-2003. Comme l'ont déjà souligné plusieurs auteurs dans ce numéro, le $3^{\mathrm{e}}$ appel à propositions pour le développement de campus numériques ne privilégie plus seulement l'enseignement à distance et la dimension pédagogique du fait qu'il se double d'un $1^{\mathrm{er}}$ appel à proposition pour la création des UNR (Universités Numériques Régionales) dont l'objectif est de proposer de nouveaux services accessibles au travers d'un dispositif technique global au sein des établissements ${ }^{6}$. Du point de vue qui nous préoccupe, alors qu'il était prévu d'engager, avec les équipes impliquées dans les 77 campus soutenus depuis 2 ans, une évaluation formative, la politique d'accompagnement se recentre sur l'évaluation de nature plutôt sommative confiée au cabinet IPSOS qui listera une série de recommandations à l'intention du commanditaire et des acteurs ${ }^{7}$. Par ailleurs, la nouvelle Ministre de la Recherche issue du changement gouvernemental du printemps 2002 s'appuiera fortement sur cette étude pour mettre en exergue un « modèle de réussite » à ses yeux, celui de l'UMVF (Université Médicale Virtuelle Francophone), préfigurateur de la nouvelle orientation politique forte vers des Universités Nationales Thématiques (UNT) qui va prendre le relais de la politique publique "campus numériques ». Assez paradoxalement - et indirectement bien sûr l'évaluation a donc contribué à la disparition de ces objets, sinon du paysage de la formation, en tous cas du paysage politique. 


$$
\text { de réussite (ou d'échec) : elles sont de l'ordre du construit social, dépendant du point de }
$$
vue adopté et des intérêts de l'acteur qui le porte.

\section{Une démarche de recherche-formation liée à une volonté d'innovation : le Campus Virtuel Suisse}

15 Le programme "Campus Virtuel Suisse (CVS) », initié par le gouvernement fédéral à la même période que le programme «Campus numériques » français (phase 1 en 2000-2003) et selon un modèle similaire de collaboration inter-établissements, a subventionné 50 projets de développement de cours en ligne au sein des hautes écoles. Ce même programme a confié une tâche de soutien et d'évaluation pédagogique à différentes unités d'enseignants-chercheurs, dont l'unité TECFA (Technologie de la Formation et de l'Apprentissage) de Genève qui a réalisé cette tâche pour 16 projets romands's.

Les responsables de ce programme d'évaluation expliquent comment ils se sont trouvés placés devant une première demande d'accompagnement des projets et d'évaluation sommative de la part de leur commanditaire, ce qui leur a paru inconciliable et contradictoire. En effet, accompagner des projets innovants signifiait pour eux adopter une démarche participative "dans laquelle tous les acteurs sont associés activement à la définition des objectifs et des stratégies qui seront mises en cuvre » et qui, en outre, ferait place à une " composante d'évaluation formative ".

17 On voit donc bien, dans ce second cas, comment les acteurs de l'évaluation ont en fait négocié le sens et la démarche d'évaluation propre à un mode d'enseignement innovateur - une recherche-action - à partir de leur vision propre de ce que peut être le pilotage de l'innovation.

18 D'un point de vue concret, ils ont procédé en début d'étude, un peu trop tardivement à leur goût par rapport au démarrage des projets (décalage dans certains cas de 6 à 18 mois), à des entretiens, assortis d'un questionnaire, avec les principaux acteurs de chaque projet (professeur responsable, coordonnateur, et quelques professeurs collaborateurs, assistants et développeurs). Ils ont par ailleurs assuré le soutien en co-organisant et en co-gérant deux séminaires d'une journée avec les acteurs de terrain, ceux-ci bénéficiant d'une demi-journée pour échanger entre eux sur leur projet respectif $9^{9}$.

Ce que nous avons retenu de leurs conclusions en regard de nos préoccupations dans cet article, c'est la façon dont l'approche méthodologique qu'ils ont adoptée, en n'isolant ni les différentes dimensions (évaluation, recherche, action, formation), ni les différentes catégories d'acteurs, les amène à déplacer les notions de succès et d'échec: "Si l'on ne prenait en compte dans l'évaluation des projets du CVS que les seuls dispositifs de formation mis en place, on serait tenté de croire à un échec relatif du programme. Et pourtant, les avancées sont importantes et témoignent du développement d'une culture du e-Learning, de l'évolution (d'un enrichissement) des représentations, des habiletés des attitudes et des pratiques qui s'y rattachent. Ces avancées pourront par la suite être réinvesties lors du développement de nouveaux cours et étendues aux personnes qui collaboreront avec l'équipe » (p. 1, draft 2004, cf. note 3).

La dynamique enclenchée sur le terrain dans la durée du point de vue de l'évolution des représentations et pratiques des acteurs leur a permis de contribuer à ce qui leur paraît être un facteur de succès privilégié dans le long terme. 


\section{La démarche de l'ERTE « Modèles économiques et enjeux organisationnels des campus numériques »} en perspective avec les deux premières démarches.

\section{Un projet de recherche académique classique}

Nous avons réalisé notre étude dans le cadre d'une Équipe de Recherche en Technologie pour l'éducation reconnue par le MEN en 2003 et financée pour les années 2003-2005. Il est important de souligner qu'il s'agit de la réponse à un appel en vue de la constitution de telles équipes de recherche associant divers labos sur la base d'un projet scientifique proposé par elles et dont des retombées sont attendues dans le champ de l'éducation. Il ne s'agit donc en aucun cas d'une commande ministérielle, mais d'un projet de recherche dont la question centrale est ainsi posée : «les trajectoires des expériences d'introduction du numérique dans l'enseignement supérieur débouchent-elles ou ont-elles en perspective un débouché sur des modèles économiques, organisationnels, institutionnels, tenables dans la durée? $»(\text { p. } 2)^{10}$. Les chercheurs concernés par l'étude sont d'origines disciplinaires différentes et mettent en œuvre des présupposés théoriques différents dans les analyses, ce qui explique pourquoi celles-ci n'ont pas été cadrées dans un format identique du point de vue de leur restitution.

\section{Un cadre institutionnel de recherche qui privilégie le rapport au terrain.}

Rappelons que la finalité que le MEN lui-même assigne aux ERTe qu'il a créées à partir de 2001 est «la résolution des problèmes liés à l'éducation et la formation. Elles se justifient par une demande de praticiens de l'éducation et de la formation ou de responsables du système éducatif confrontés à un enjeu concret ou un problème ${ }^{11}$. La demande, en ce qui nous concerne, a pris corps dans des discussions informelles entre une actrice du département TICE du MENR s'interrogeant sur les conditions de pérennisation des projets issus du programme "campus numériques» et une partie de l'équipe d'enseignants-chercheurs qui se mobilisera sur l'étude et qui l'était déjà sur le terrain pour un certain nombre d'entre eux, à titre d'évaluateurs ou à titre d'acteurs, dans des projets antérieurs aux campus. Le principe qui a réuni ces chercheurs a été de s'engager dans un véritable travail de terrain une fois réalisé l'échantillon des campus retenus, par le biais d'interviews qualitatives auprès des principaux acteurs, d'une collecte de documents, de la visite des sites correspondant aux dispositifs de formation etc., en laissant aux investigateurs une grande liberté dans la mise en œuvre de l'importante grille d'analyse bâtie en commun, ceci précisément afin de respecter au mieux la spécificité de chacun des terrains.

Bien que nous n'ayons clairement jamais eu une volonté de préconisation ou de recommandations, nous avons par ailleurs bien entendu restitué les études aux acteurs de chaque campus, sous des formes diverses, voire continué à rencontrer les acteurs dans tel ou tel type d'occasions ${ }^{12}$.

Le point fort que nous mettrons en avant cette fois par rapport à notre problématique "succès/échec ", c'est qu'une telle démarche nous permet maintenant de revenir sur l'idée de l'évaluation comme «construit social » avancée ci-dessus en conclusion de la 
première démarche d'étude et d'approfondir l'opposition avec une conception positiviste de l'évaluation. Celle-ci reposerait sur l'idée que tout peut être anticipé, calculé et réparti de manière rationnelle par rapport à un initial clairement défini en termes d'objectifs mesurables et qu'il suffirait en fin de parcours de chiffrer les réalisations pour apprécier la réussite en terme de degré de conformité par rapport aux attendus. Notre démarche de recherche s'est appuyée quant à elle sur une conception constructiviste de la dynamique de projet : certes, il s'agit bien de partir des objectifs singuliers de chaque projet et de ce qui apparaissait possible en terme de réalisation pour observer ce qu'il en est advenu dans la "fenêtre» de temps sur laquelle ouvrait notre étude; mais il nous paraissait surtout important de comprendre les éventuelles contradictions de départ dans les objectifs, les formes d'équilibre qui se sont cherchées, ce qui en a favorisé ou entravé le développement, les difficultés et les fragilités, quelles que soient les réussites effectivement constatées. Autrement dit, nous sommes bien sur l'idée que la dynamique du projet résulte de ce que les acteurs construisent socialement à partir d'un donné qui s'impose à eux (les critères de l'appel à projets, certains héritages...) et de ce qu'ils prennent l'initiative de proposer et de mettre en œuvre collectivement.

\section{Les intentions innovantes et leur évaluation}

Dans cette seconde partie, nous allons aborder plus concrètement l'analyse du matériau de terrain en procédant à un repérage des intentions innovantes des diverses catégories d'acteurs pour nous interroger sur ce qu'il en est advenu à la fin de la période d'observation, ce qui, chemin faisant, nous amènera à faire référence à des aspects abordés sous différents angles par les contributeurs dans ce numéro.

\section{Le repérage des intentions innovantes}

Parler d'intentions innovantes, c'est bien entendu se référer à des sujets porteurs d'une telle visée. Nous avons retenu de la lecture d'un auteur spécialiste de l'innovation en éducation, G. Adamczewski (1996) que celle-ci correspond à «l'émergence d'une nouveauté qui se situerait moins dans un contenu que dans le faire autrement dans un milieu donné ». Autrement dit, dans bon nombre de cas, projet et intention de changement sont portés par des acteurs engagés dans une démarche de transformation des pratiques à l'intérieur de laquelle se font des apprentissages. Il s'agit donc d'un changement plus émergent que planifié, d'« aventure sociale voulue " (et donc aussi de risque assumé), de «subjectivité dans la perception de l'innovation" (il faut bien que les acteurs de l'innovation y voient un intérêt puisque ce sont eux qui agissent) selon les formules de F. Cros (1996, pp. 43-44), ce qui nous ramène ainsi à l'idée de « construit social » évoquée plus haut.

Mais de quels acteurs est-il question, à quel niveau opèrent-ils, et quelles intentions mettent-ils en avant?

Les responsables politiques ministériels ont affiché une intention forte, traduite en critères d'éligibilité : celle de mettre en place de véritables dispositifs de formation à distance en ligne et pas seulement des ressources numérisées ${ }^{13}$ (comme le fait l'UMVF et comme prévoient de le faire les UNT...) ; et ils ont ordonné à cette fin la création de consortiums, là où prévalaient jusque là des projets isolés. Le texte de l'appel à projets justifie cette obligation par un certain nombre de considérations pratiques $^{14}$, 
recommande de s'allier au CNED et de ne pas négliger les partenaires étrangers pour favoriser une stratégie internationale.

Tel est le « donné » ou le cadre de contraintes et d'opportunités qui s'offre aux acteurs de terrain, pour bon nombre déjà (en particulier ceux qui assureront le rôle de responsable de projets) engagés de longue date dans des expériences liées aux TICE. Ils vont s'en servir pour leurs propres intentions, qui sont d'innover pédagogiquement en travaillant, comme la contractualisation avec le MEN le leur demande, l'ingénierie de formation (mettre sur pied les dispositifs en pensant contenu, accompagnement et logistique adéquate) et l'ingénierie de ressources. Secondairement, il leur faut aussi innover dans le management de projet à plusieurs et réussir les partenariats, ce qui situe cette fois le problème de l'évaluation au méso-niveau de l'organisation intra et inter-établissements et non plus seulement au micro-niveau des dispositifs médiatisés de formation.

\section{Dimensions évaluatives}

\section{Les acteurs ont effectivement produit des dispositifs de formation...}

C'est un fait: les consortiums étudiés ont mis sur pied des dispositifs de formation qui articulent le plus souvent Formation Initiale/Formation Continue/Formation A Distance, dans des temps plutôt record pour certains (deux ans pour CampusCultura malgré de nombreuses difficultés). Comme l'ont montré ici même F. Horn et T. Lamarche, il y a le cas singulier à l'époque de l'UMVF (l'Université Médicale Virtuelle Francophone) dont la visée est celle d'un métacampus proposant un ensemble de ressources hétérogènes produites en dehors du Groupement d'Intérêt Public qui a constitué son cadre organisationnel. Mais, dans les autres cas, les initiateurs des campus se sont centrés sur l'organisation ou la réorganisation globales de dispositifs pédagogiques, deux tendances qu'il ne faut pas bien sûr rigidifier - apparaissant : l'une est que les porteurs de projets sont partis d'usages précis sur lesquels étaient effectivement, dès le départ, engagés des enseignants. C'est le cas de Forse ou de CampusCultura, qui avaient monté les formations soit en mode présentiel, soit en mode à distance sans recours aux outils numériques et les offraient déjà à des publics étudiants. Ce n'est que progressivement qu'ils ont construit des outils et des ressources numériques, avec des différences dans le rythme ${ }^{15}$. L'autre tendance recouvre le fait que les initiateurs de projets, tout en ne retardant pas les formations à mettre sur pied, ont privilégié malgré tout la production des ressources numérisées en investissant des sommes importantes qui ont pu grever ensuite la mise en place des services d'accompagnement par le biais du tutorat. Le campus Canège est celui qui illustre le plus clairement cette autre tendance, le quatrième, C@mpusciences ayant lui aussi une caractéristique particulière puisque l'engagement par les ressources avait été le fait des acteurs du projet dont il est issu, l'Université en ligne (UeL), et que le campus a précisément été labellisé pour assurer une diffusion et des usages à ces ressources.

Donc, la mise en œuvre de dispositifs de formation a été tenue même si cet objectif s'est réalisé selon des modes d'engagement qui marquent de leur empreinte le devenir du projet. 
...mais qui n'ont pas tous connu la même réussite en termes d'effectifs

A présent, si l'on s'interroge sur l'efficacité des formations en termes de nombre d'inscrits et de nombre de diplômés, c'est sans doute là que les faiblesses apparaissent le plus. Ainsi, un des campus, Canège, offre-t-il un exemple de disproportion particulièrement forte entre les objectifs ambitieux annoncés en termes d'effectifs et le nombre très faible d'inscrits au bout de 2 ans d'existence ${ }^{16}$. Un autre campus, Forse, offre un exemple contraire en ayant quantitativement et qualitativement atteint et même dépassé les objectifs qu'il s'était fixés ${ }^{17}$. Mais globalement, les montées en charge correspondent rarement aux ambitions de départ, les conditions d'effectuation de cellesci restant la rencontre avec une demande importante de formation à distance diplômante solvable et la recherche d'une répartition équilibrée des moyens entre ressources et service de tutorat.

\section{La production des ressources a été effective au sein des consortiums, assez peu réellement collaborative et peu standardisée}

Les campus se sont organisés selon des modalités différentes, elles-mêmes reliées à des représentations et des intérêts qui n'étaient pas les mêmes, en ce qui concerne l'ingénierie de ressources, comme en témoigne ici-même l'article de Y. Combès et $\mathrm{A}$. Bal. Un autre paramètre très important est le degré de sophistication engagé dans les divers types de réalisations multimédia qui implique des expertises différentes en terme de métiers dès qu'il est élevé. Nous nous contenterons de souligner deux traits que nos études font émerger :

- Il y a eu peu de démarche collective dans l'ingénierie pédagogique et un certain manque de vision d'ensemble, les décisions se prenant formation par formation au niveau des équipes responsables, comme dans Canège et Forse. L'explication vient sans doute du fait qu'a prévalu une logique de partage des cours entre les équipes liées à des diplômes, chacun restant maître de son domaine d'expertise dans le cours ou le module dont il a la responsabilité (lequel, relevant le plus souvent de son domaine d'enseignement en présentiel, avait déjà été réalisé dans ce cadre statutaire). CampusCultura, a contrario, a cherché à définir les ressources à produire par module de manière centralisée, à partir de l'ensemble du budget disponible, mais cette procédure était sans doute plus facile pour ce campus du fait qu'il était centré sur un seul diplôme ;

- Même dans le cas où des professionnels de la scénarisation multimédia sont intervenus, la séparation des tâches avec les auteurs de cours est restée relative, comme le montre le cas de CampusCultura (E. Fichez et A. Benchenna, 2005, p. 19). Et, dans les deux campus numériques où des ressources ont été produites en nombre (Canège et CampusCultura), les contraintes liées aux normes et aux standards sont restées faibles, faisant surtout l'objet de souhaits laissés à l'appréciation et à la responsabilité des enseignants-auteurs. Il faut toutefois mettre un peu à part l'un des campus, C@mpusciences pour lequel la problématique des standards a eu beaucoup d'importance du fait de sa trajectoire propre.

\section{...et a conduit à des apprentissages en termes de e-learning}

Toutefois, si les acteurs enseignants n'ont pas perçu dans l'ensemble de rupture forte concernant l'évolution de leurs tâches par rapport aux dispositifs classiques, ils se sont malgré tout trouvés confrontés à un changement de statut du cours, celui-ci nécessitant, 
en amont de l'acte de diffusion, un travail de conception et de rédaction complète, auquel ils sont très peu habitués. Julien Deceuninck (2005, note 13, p. 25) parle ainsi « de formes différentes d'enregistrement social d'un acte culturel: d'un côté, la matérialisation se traduit par une reconnaissance des droits dans le temps (ici trois ans); de l'autre, l'enseignant, par la non inscription sur un support, garde personnellement la maîtrise de son cours à travers la possibilité de répétition de sa prestation face aux étudiants $\%$. On a donc ici une illustration de ce que les acteurs apprennent du point de vue de la culture du e-learning, qui peut être, comme le soulignait $\mathrm{D}$. Peraya, un facteur de succès à plus long terme que les produits réalisés en eux-mêmes.

\section{La réussite non prévue de la dimension communicationnelle pour les apprenants} CampusCultura ${ }^{18}$, tient aux dynamiques communicationnelles d'apprentissage qui se sont développées au sein des groupes de formés grâce aux outils qu'offraient les plates-formes et bien entendu aussi grâce à l'animation tutorale. Comme le soulignent deux auteurs d'une évaluation externe centrée sur les usagers de CampusCultura, "La plate-forme est également un lieu où les étudiants mettent en commun les multiples composantes de leur expérience individuelle d'apprentissage, dans ses aspects didactiques mais aussi dans ses dimensions plus psychologiques et plus personnelles. Le forum général de CampusCultura, très actif, est visité de façon régulière par une majorité d'étudiants qui contribuent peu mais se disent très attachés à sa fonction de mutualisation des pratiques car ils en retirent des bénéfices, notamment pour l'entretien de leur motivation. C'est un espace communautaire où chacun peut faire part de ses difficultés et recevoir des messages d'encouragement $»^{19}$.

Quant à P. Grevet, dégageant quelques traits du tutorat effectivement mis en œuvre dans Canége, il relève trois catégories de contenu apparaissant dans les messages compilés sur les forums de la plate-forme pendant une période de deux mois en 2005: «La première relève de la socialisation élémentaire dans un groupe d'apprenants avec des échanges divers d'informations y compris des nouvelles personnelles... La seconde correspond à des entraides entre étudiants, ou à des demandes d'intervention adressées aux enseignants, pour résoudre des difficultés techniques rencontrées dans l'accès aux ressources... La troisième catégorie a trait aux apprentissages proprement dits» (2005, p. 47). Et l'auteur de conclure, en s'appuyant sur l'ensemble des messages, que ceux-ci "témoignent... de la possibilité de générer avec le numérique et Internet une dynamique de groupe d'apprentissage à distance » (p. 48).

\section{L'organisation en consortiums : des signes de décomposition...}

Qu'en a-t-il été, dans les faits, des dynamiques partenariales ${ }^{20}$ ?

Certes, elles ont réussi à se mettre en place, les chefs de projet (le plus souvent des enseignants-chercheurs impliqués de longue date dans le domaine des pratiques innovantes) ayant réussi un positionnement fort leur permettant d'obtenir des engagements appréciables de leur propre établissement et de nouer des collaborations inter-établissements. Mais les campus n'existent qu'à travers les filtres classiques qui sectorisent les fonctionnements universitaires : imbrications de cultures (grades, statuts, pratiques) et de structures différentes liées à la cohabitation entre trois secteurs (FI, FC, FAD) qui ont leur mode de financement propre souvent lié à la maîtrise des publics locaux ou nationaux. La conséquence de cet état de fait est la menace présente presque partout de risques de décomposition par velléités de ré-autonomisation des établissements. Un 
rapide bilan des situations à la fin de la période d'observation confirme que Forse n'est pas seul à connaître des tentations de recentrage en région et d'autonomisation : Canège manifeste aussi de son côté une tendance à la contraction institutionnelle ${ }^{21}$. On constate ainsi dans ce consortium soit la réappropriation de certains diplômes par une université seule, soit la mise en place de 3 offres distinctes concernant la même année de formation en concurrence interne (à la rentrée 2005). Quant à CampusCultura, qui a connu deux crises successives aboutissant chaque fois à des départs, voire à ce qui a été ressenti comme des exclusions, il semblerait aujourd'hui en voie d'être maintenu en fonctionnement par un seul établissement, celui de l'Université d'Aix-Marseille, après une série de changements de responsables au CNED de Vanves et le désengagement au final de celui-ci.

De fait, dans bien des cas, l'établissement du porteur de projet reste en situation prépondérante, car les campus, faute d'une institutionnalisation qui les aurait confortés, sont restés dans une logique de dépendance vis-à-vis de leurs porteurs, comme le souligne J. Deceuninck pour Forse, mais qui pourrait aussi bien être repris pour d'autres campus: "Aucune institutionnalisation n'est venue conforter la situation initiale de l'équipe projet et le campus reste soumis au risque d'une décomposition du réseau qui le sous-tend, que celle-ci vienne des instances de direction des établissements partenaires (Universités, CNED) ou d'un désengagement d'un ou de plusieurs de ses chefs de projet initiaux. Malgré sa relative durée, Forse reste encore aujourd'hui un dispositif que l'on peut qualifier de 'dépendant de ses porteurs' par opposition aux dispositifs institutionnels » (Deceuninck, 2005, p. 44).

\section{...et de recompositions}

Mais, de la même façon que les consortiums ne sont pas des objets créés ex nihilo au moment de l'appel à projets et se sont appuyés sur des réseaux d'acteurs ou des projets antérieurs $^{22}$, les décompositions actuelles ne sont pas synonymes de disparition ni même d'éclatement programmés. Le modèle d'un portail de diffusion libre des ressources « inventé » par l'UMVF sous la forme plus institutionnalisée d'un GIP ayant vocation à rassembler nationalement toutes les universités de la discipline médicale, semble une voie de recomposition que valorise aujourd'hui la politique ministérielle en faveur des UNT affichée depuis le colloque de Montpellier de l'automne 2003 et publicisée à travers une manifestation très officielle en mai $2006^{23}$ : au modèle du «campus numérique » s'est substitué celui de l'Université Numérique Thématique (UNT) ${ }^{24}$, un virage que certains de nos campus sont prêts à prendre, tel Canège dont le porteur de projet M. Armatte, œuvre à présent dans le cadre de l'UNT Aunège (pôle d'excellence en économie-gestion) annoncée comme l'une des cinq déjà en place parmi les 10 prévues pour 2007, pour un public de 160000 étudiants potentiels ${ }^{25}$. C@mpusciences de son côté se tourne aussi vers les UNT auxquelles il manquerait les sciences fondamentales et que l'Université en ligne (Uel) pourrait initier avec des contenus déjà évolués.

\section{Conclusion}

$\mathrm{Au}$ terme de cette réflexion, nous résumerons quelques points saillants en réponse à la question posée au départ de la pertinence des notions de réussite ou d'échec des CNF et nous relancerons le débat en posant quelques questions pour l'avenir. 
43 En premier lieu, nous confirmons, s'il en était besoin, qu'il n'y a pas de réussite ou d'échec en soi ou globale des campus. Il y a réussite ou échec selon le point de vue adopté et la réalité sociale qu'il construit : un projet, l'UMVF, a ainsi constitué par tâtonnement le creuset d'un modèle fortement et officiellement reconnu aujourd'hui (celui de l'édition numérique de contenus pour l'enseignement à travers les UNT), tandis qu'un autre, C@mpuSciences, comme le montre P. Moeglin, en constitue un autre encore à peine identifié (ingénierie de médiation, courtage), mais dont l'auteur pense qu'il pourrait avoir un avenir. D'autres projets - Canège, Cultura, mais aussi C@mpuSciences si on le considère sous un autre angle - présentent, à des degrés divers, et selon ce qui est pris en considération, des éléments de réussite (l'expérience d'innovation pédagogique pour les enseignants, de parcours pédagogique plus souple et plus riche pour les étudiants, de maillage entre partenaires pour les porteurs de projet...) et des éléments d'échec (déséquilibre financier pour les gestionnaires, décomposition des partenariats...).

La nature des projets à évaluer est un premier paramètre important dont il faut tenir compte : il s'agit ici de projets en incubation pour lesquels l'appel ministériel a joué un rôle d'impulsion. Claude Lépineux, Responsable du développement dans une association de conseil en formation et représentant du secteur privé dans les jurys des trois appels à projets, rappelle dans un entretien avec L. Petit "qu'il faut accepter de jouer le rôle d'impulseur de projets pour que des initiatives se mettent en place. C'est exactement la même chose que pour les capitaux à risques: une manière d'impulser en acceptant la perte» (2006, p. 95). Qu'il y ait de la déperdition, des échecs dans ce que nous avons été étudié, ou globalement dans les campus, n'est donc pas en soi étonnant ${ }^{26}$.

Le contexte d'évaluation est un second paramètre important: les projets doivent être resitués dans une temporalité plus étendue que celle qui coöncide avec leur définition stricte, autant en amont qu'en aval, et par conséquent être resitués aussi dans les politiques publiques qui permettent de comprendre leur trajectoire.

Enfin, dynamique de projet et dynamique d'évaluation vont de pair : les conditions de réussite sont liées à la capacité des acteurs à faire évoluer leur projet de manière réaliste et pragmatique, souvent en s'adaptant aux possibilités de financement: les exemples contrastés de Canège et de Forse montrent que les projets qui semblaient destinés à une grande réussite ne sont pas nécessairement ceux qui tiennent leurs promesses. En conséquence, l'évaluation doit tenir compte de ce caractère évolutif et se faire, non seulement en coupe transversale pour observer des résultats chiffrés à un temps " $\mathrm{t}$ " donné, mais aussi dans la diachronie pour identifier les moments-clefs de la dynamique d'adaptation, les bifurcations, etc.

Concernant les questions, nous en soulèverons deux séries, liées en fait aux deux intentions innovantes que nous avons identifiées chez les acteurs.

Pourquoi globalement si peu d'engagement des universités constitutives des consortiums dans l'institutionnalisation des projets? Pourquoi des forces autocentrées sur chaque université continuent-elles à jouer? Se sont-elles interrogées sur la place et le sens qu'elles donnaient à ces projets en regard de leurs missions ou ont-elles considéré que ceux-ci restaient surtout l'affaire de leur porteur principal ? Corollairement, pourquoi le regroupement sur une base disciplinaire dans les grands conglomérats que seront sans doute les UNT susciterait-il un attrait plus fort et ne rencontrerait-il pas les mêmes difficultés? 
Et pourquoi, après les leçons tirées semble-t-il de l'expérience de l'Uel (ne pas dissocier production des ressources et usages de celles-ci) et après l'insistance mise sur le critère d'éligibilité des projets CNF (proposer des dispositifs de formation), voit-on revenir en force sur le devant de la scène un modèle, les UNT, qui fait l'impasse sur ces principes? Est-ce la difficulté de l'entreprise qui est en cause ? Ou est-ce son évitement dans les UNT, au profit d'un renvoi hypothétique vers les établissements de base censés mettre en usage les ressources dans les dispositifs de formation, qui ferait de ce nouveau modèle... le succès du moment?

\section{BIBLIOGRAPHIE}

Adamczewski, G., (1996), «La notion d'innovation : figures majeures et métaphores oubliées », in : F. Cros et G. Adamczewski (éds.), L'innovation en éducation et en formation, De Boeck INRP.

Combès, Y. et Moeglin, P., (2005), « C@mpuSciences, d'un modèle industriel à l'autre », accessible aux adresses indiquées à ERTe CN 2005.

Cros, F. et Adamczewski, G., (éds.), (1996), L'innovation en éducation et en formation, De Boeck INRP.

Delaunay-Jacquinot, G., (2008), « Introduction », in : Jacquinot-Delaunay G. et Fichez E., (sous la dir. de), Université et TICE : chronique d'une innovation annoncée, De Boeck Université.

Deceuninck, J., (2005), « Campus Forse. Formation et ressources en sciences de l'éducation », accessible aux adresses indiquées à ERTe CN 2005.

Dupuis, C., Schmitt, C., Galissi, V. et Robion, B., (2003), Évaluation globale des campus numériques, Méthodologie et questionnaires, SFRS/Ipsos, ftp://trf.education.gouv.fr/pub/educnet/ chrgt/methodologie.pdf.

ERTe CN, (2005), Résultats 2005 de l'ERTe (Équipe de Recherche Technologique éducation) « Modèles économiques et enjeux organisationnels des campus numériques », résultats accessibles à www.ifresi.univ-lille1.fr/SITE/2_Recherche/22_Programmes/ERTe/ERTe.htm ou à partir de la page d'accueil www.mshparisnord.org/.

Fichez, E., (2006a), Actes du colloque TICE Méditerranée 26-27 mai 2006, Gênes, accessible à partir du site : http://isdm.univ-tln.fr.

Fichez, E., (2006b), « Campus numériques : des ambitions à l'épreuve des terrains », in : Distances et Savoirs, vol. 4, nº 3/2006, Hermès-Lavoisier.

Fichez, E. et Benchenna, A., (2005), « Le cas CampusCultura », accessible aux adresses indiquées à ERTe CN 2005.

Grevet, P., (2005), « L'expérience socio-économique de Canège », accessible aux adresses indiquées à ERTe CN 2005.

Horn, F. et Lamarche, T., (2005), « UMVF », accessible aux adresses indiquées à ERTe CN 2005.

Lépineux, C., (interview par L. Petit), (2006), « Entretien avec Claude Lépineux responsable du développement chez Algora », Distances et Savoirs, vol. 4, n 1/2006, Hermès-Lavoisier, 93-99. 
Pouts-Lajus, S. et Lecchia, E., (2004), «Évaluation formative accompagnant la mise en place de CampusCultura (CNED). Usages et usagers », Éducation et Territoires, Rapport, 35 pages.

Thibault, F., (2006), « Autour des campus numériques français. Repères dans les initiatives du ministère en charge de l'enseignement supérieur ", Distances et Savoirs, vol. 4, n 1/2006, HermèsLavoisier, 109-112.

Viens, J. et Peraya, D., (2004), Une démarche de recherche-action de type Évaluation-formation pour soutenir l'innovation pédagogique en e-learning, in : J. Viens \& A. Wyrsch (éds.), Revue suisse des sciences de l'éducation (Bilan et perspectives : rôle, approches méthodologiques et impacts de l'évaluation sur la qualité pédagogique de cours e-learning en processus de développement/ implantation. Numéro Thématique), 2, 229-249.

\section{NOTES}

1. Ce questionnement a fait pour nous l'objet d'une première réflexion dans une communication proposée à Gênes ( $c f$. E. Fichez, 2006a).

2. Partant de cette position, nous avons fait le choix dans cet article de ne faire porter la réflexion que sur les 5 campus français étudiés dans le cadre de l'ERTE, les études canadienne et québécoise relevant d'un contexte politique trop différent du contexte français. Pour une mise en perspective des expériences françaises avec celles-ci, voir E. Fichez, (2006b).

3. Cette citation est extraite d'un draft intitulé "Démarche de recherche-action de type Évaluation-formation pour soutenir l'innovation pédagogique en e-learning » (pp. 9-10). Ce prépapier (non consultable désormais) a donné lieu à une publication (Viens et Peraya, 2004).

4. Il semble, d'après l'enquête IPSOS à laquelle nous allons faire référence, que $80 \%$ des « campus numériques » français avaient des antécédents sous une autre appellation avant d'être reconnus comme tels ou labellisés.

5. "Éducation et Territoires » a réalisé deux évaluations pour le compte du porteur de projet CampusCultura, l'une sur le processus de production des ressources et l'autre sur les usages et usagers du campus (cf. Pouts-Lajus et Lecchia, 2004).

6. Ces UNR s'appuient sur les quatre projets d'Environnements Numériques de Travail (ENT) retenus en 2002 : ESUP-Portail ; ENCORA ; EPPUN ; Monte-Cristo (cf. F. Thibault, 2006, p. 110).

7. Le projet d'évaluation formative prévu pour 2002 est évoqué par F. Thibault (2006, p. 110). L'étude sommative finalement retenue conclut que le programme dans son ensemble satisfait les attentes bien que son impact soit limité. Six conditions nécessaires à la pérennité des projets sont posées (Cf. Résultats de l'évaluation globale des campus numériques: http:// www2.educnet.education.fr/sections/superieur/campus/evaluation/).

8. Nous avons choisi ici d'évoquer l'expérience suisse parce qu'elle concerne un objet proche des campus numériques français, mais nous aurions pu tout aussi bien évoquer la démarche de recherche-action duCODIF (Comité pour l'Observation et l'Orientation des Dispositifs de Formation) à propos de l'entreprise RUCA/UeL/C@mpusciences dont G. Jacquinot-Delaunay rappelle ainsi les caractéristiques : "il s'agit bien de présenter les résultats d'une recherche, menée entre fin 1999 et juin 2004, par un groupe interuniversitaire et interdiciplinaire composé d'enseignantschercheurs de disciplines scientifiques (informatique, mathématiques, physique, chimie, biologie) et de sciences humaines, principalement des sciences de l'éducation et des sciences de l'information et de la communication - chose assez rare pour être soulignée. Tous enseignants et chercheurs dans leur discipline d'origine, ils se retrouvaient pour l'occasion, les premiers hommes et femmes de terrain, les seconds, experts ou chercheurs dans le champ des innovations pédagogiques liées aux technologies " (Introduction, in : Jacquinot-Delaunay G. et Fichez E., à paraître en 2008). 
9. Deux thématiques ont fait l'objet des séminaires : "Innovation et e-Learning : vers une pédagogie active » et "Évaluation, Innovation et e-Learning: pour une évaluation des aspects pédagogiques innovateurs des projets CVS et de leurs impacts sur les enseignants et les apprenants ". Pour plus d'information sur ces séminaires, consulter : http://tecfa.unige.ch/proj/cvs/seminar.html.

10. Cf. Présentation en ligne de l'étude ERTe $\mathrm{CN}$, Résultats 2005 de l'ERTe (Équipe de Recherche Technologique éducation) accessibles à partir de la page d'accueil www.mshparisnord.org/. Les citations de ce paragraphe sont extraites de cette Présentation.

11. Cf. Présentation des Erte sur le site du gouvernement à l'adresse http:// www.education.gouv.fr/discours/2001/dprost.htm\#2 (ERTE).

12. Nous avons ainsi dialogué, à Marseille, lors des Journées de l'Orme en mars 2006, avec le porteur du campus Forse à Rouen (J. Wallet) et avec J. L. Faure, directeur du CNED-EIFAD à Poitiers. P. Grevet a maintenu des relations régulières avec M. Armatte, le porteur du campus Canège. Nous-même avons été amenée à tirer parti des résultats à la demande de responsables universitaires palestiniens de projets FOAD (particulièrement intéressés par la question des conventions ou des chartes)...

13. Rappelons les trois critères d'éligibilité des appels à projets : mettre sur pied des dispositifs de formation; produire et/ou introduire des ressources numérisées; assurer la logistique pédagogique et administrative par le biais des plates-formes (Appel à projets, juin 2000).

14. " [La constitution d'une offre nationale à distance] dépasse les possibilités d'un seul établissement. Elle nécessite des collaborations et des financements importants, non seulement pour les contenus de formation, mais aussi pour la qualité et la pertinence de leur mode de diffusion, pour la mise en place des services d'accueil et de tutorat à distance et pour les facilités nécessaires au regroupement des publics en mode présentiel pendant de courtes périodes " (Appel à projets, juin 2000, p. 2).

15. Les acteurs de CampusCultura voulaient précisément se démarquer de la formation existante (la licence «Conception et Management de Produits Culturels») par la production et la mise en usage rapide de nouvelles ressources et de nouveaux services, tandis que Forse y est allé sans hâte, privilégiant dans une première phase des techniques classiques (envoi de papiers, de cassettes, etc.). J. Wallet, porteur du projet Forse, lors de l'échange à Marseille (Journées de l'Orme, mars 2006) a indiqué que la fabrication de ressources numériques se développait maintenant à un rythme plus soutenu. On se reportera ici même aux articles d'A. Benchenna et de V. Brulois ainsi que de J. Deceuninck pour plus de détails sur ces campus.

16. Les effectifs étudiants étaient en effet de l'ordre de 200 en 2003-2004 contre 1820 prévus pour cette même année dans le dossier déposé par Canège en réponse à l'appel à projets 2001. En 2004-2005, les effectifs se seraient encore réduits du fait des entrées moins nombreuses en première année du DEUG.

17. Le campus Forse (sciences de l'éducation) compte plus de 1000 étudiants au total en 2004-2005, ce qui constitue une performance au regard des résultats de nombreux autres campus. L'effectif en troisième cycle est resté stable depuis le départ et celui de la licence a été en croissance (source : page d'accueil du campus à l'adresse http://www.sciencedu.org/forseaccueil/ index.htm?PAGE=Ressources/Partenaires/URouen.htm).

18. L'UMVF est, on l'a dit, à part et ne se soucie guère des usages étudiants. L'étude «C@mpusciences» n'aborde pas ce point. En ce qui concerne Forse, son responsable, J. Wallet, évoquait lors des Journées de l'orme (mars 2006) un changement de pratiques communicationnelles étudiantes se manifestant par une génération de bloggers, qui n'hésitent pas à créer des sites parallèles.

19. S. Pouts-Lajus et Elisia Leccia, op. cit., septembre 2004, p. 33.

20. Nous serons assez brève que ce point qui a fait l'objet de développements plus approfondis dans notre article de Distances et Savoirs (Fichez, 2006b).

21. On se reportera pour plus d'explications à P. Grevet, 2005, pp. 62 sqq. 
22. Le réseau Cultura réunissait ses membres sur une base à la fois professionnelle et militante depuis les années 90 autour de la licence CMPC qui existait à distance de façon classique ; Canège s'inscrit dans des trajectoires de politiques, d'institutions, et d'acteurs, entamées antérieurement par les six établissements qu'il rassemblera une fois constitué, etc.

23. Il s'agit du Colloque International «L'Université à l'ère du numérique » (CIUEN), www.ciuen.org organisé les 22-23-24 mai 2006 à la Cité des Sciences et de l'Industrie de la Villette à Paris par le Professeur Benhamou, Directeur de l'UMVF, sous l'égide du MEN.

24. Ces UNT, dont 5 ont été mises en place en 2005 et 10 sont prévues pour 2007, sont définies par F. Thibault (2006) «comme des organismes 'sans murs' fédérant des grands groupes de disciplines et rassemblant potentiellement tous les établissements supérieurs concernés (disciplines juridiques, technologiques, économie/gestion...) afin de mettre à disposition des ressources pédagogiques numérisées ». 25. Source : Bilan du déploiement des UNT http://www2.educnet.education.fr/sections/superieur/ unt/bilan_du_deploiement.

26. F. Thibault, en mars 2006 aux Journées de l'Orme, faisait remarquer qu'on trouve aujourd'hui encore la trace de 44 d'entre eux sur 77.

\section{RÉSUMÉS}

L'auteure s'intéresse dans cet article à la dimension évaluative des campus numériques étudiés dans l'ERTe en se demandant si les notions de succès et d'échec sont pertinentes à poser telles quelles à leur propos. Ceci l'amène dans un premier temps à problématiser trois exemples de démarches méthodologiques en usage pour de tels objets. Puis, définissant les campus comme des "projets de dispositifs de formation médiatisés liés à des intentions innovantes ", elle en retient quatre principales, dont trois explicitement attendues (la production de dispositifs de formation; la production des ressources au sein des consortiums ; l'organisation en consortiums) et une non formulée (la dimension communicationnelle pour les apprenants) et fait le point sur ce qu'il lui semble en être advenu au terme d'une période limitée d'observation (2003-2005) en termes de tendances plus que de réalités stabilisées. Elle revient en conclusion sur la pertinence des notions évaluatives en jeu.

This author examines the evaluative dimension of the virtual campuses studied in the ERTe and questions whether the success and failure notions are relevant. First, this leads to problematize three examples of methodological strategies utilized in these projects. Then, defining virtual campuses as "projects which create mediatised educational systems and that are tied to long term innovative strategies ", the author focuses on four principal objectives, three of which are explicitly stated (the production of educational systems; the production of educational ressources within a consortium, the organisation of the consortium), while one which is not formulated (the communicational dimension for learners) and resumes what has occurred to these objectives at the end a limited period of observation (2003-2005) more as tendencies than stabilised realities. To conclude, the author requestions the relevency of the evaluative notions put forth in the projects. 
INDEX

Keywords : training, innovation, education, success, failure, digital campus, France, research Mots-clés : formation, innovation, évaluation, succès, échec, campus numérique, France, recherche

\section{AUTEUR}

\section{ÉLISABETH FICHEZ}

Élisabeth Fichez est professeur émérite à l'Université Lille 3, attachée au laboratoire GERIICO et membre du Séminaire Industrialisation de la Formation. Ses travaux de recherche portent sur des dispositifs de formation intégrant le numérique dans l'enseignement supérieur (campus numériques français et étrangers, pratiques collaboratives d'apprentissage par Internet, etc.). Outre la coordination de publications issues de ces deux terrains de recherche, elle co-dirige avec G. Jacquinot-Delaunay un ouvrage à paraître prochainement : «L'université et les TIC : chronique d'une innovation annoncée ». Adresse électronique : elisabeth.fichez-vallez@univ-lille3.fr 